\title{
Electron Shape and Structure: A New Vortex Theory
}

\author{
Nader Butto \\ Petah Tikva, Israel \\ Email: nader.butto@gmail.com
}

How to cite this paper: Butto, N. (2020) Electron Shape and Structure: A New Vortex Theory. Journal of High Energy Physics, Gravitation and Cosmology, 6, 340-352. https://doi.org/10.4236/jhepgc.2020.63027

Received: May 19, 2020

Accepted: June 29, 2020

Published: July 2, 2020

Copyright $\odot 2020$ by author(s) and Scientific Research Publishing Inc. This work is licensed under the Creative Commons Attribution International License (CC BY 4.0).

http://creativecommons.org/licenses/by/4.0/

\begin{abstract}
Along with all other quantum objects, an electron is partly a wave and partly a particle. The corpuscular properties of a particle are demonstrated when it is shown to have a localized position in space along its trajectory at any given moment. When an electron looks more like a particle it has no shape, "point particle", according to the Standard Model, meaning that it interacts as if it is entirely located at a single point in space and does not spread out to fill a three-dimensional volume. Therefore, in the sense of particle-like interactions, an electron has no shape. In this paper, a new theory is proposed in which the electron has a structure and a shape. The central idea is that an electron is a frictionless vortex with conserved momentum made out of condensed vacuum generated in the Big Bang from massless virtual photons that acquire mass when moving in the vortex at the speed of light. Using Hydrodynamics laws and applying them on the superfluid vacuum the basic properties of the electron are described here forth. This study provides mathematical models to calculate the mass, kinetic energy, density, volume, time, charge, and particle-wave duality. Such mathematical formulations are presented to confirm the theory. We conclude that the shape of the electron is accessible to human imagination, knowing its shape helps to determine its properties and shed a light on how matter is made and to explain the interactions of sub-atomic particles.
\end{abstract}

\section{Keywords}

Electron Structure, Vortex, Hydrodynamics Laws, Vacuum Density

\section{Introduction}

The electron is a fundamental particle of nature, is the essential constituent of electric currents, and together with protons and neutrons, is the most important 
element of an atom's structure. It is negatively charged and may be free or bound to an atom in electronic orbits. The electrons are subatomic particles, play an essential role in numerous physical phenomena, such as electricity, magnetism, chemistry and thermal conductivity, and they also participate in gravitational, electromagnetic and weak interactions.

According to current experiments and theories, the electron is a structure-less, point-like object, made out of nothing [1], and its entire mass is concentrated in its extension-less center.

An electron looks like a particle when it interacts with other objects in certain ways (such as in high-speed collisions) and is not point-like, as stated by quantum mechanics.

In quantum mechanics, depending on the observation point of an electron, it can appear to be a particle or it can appear to be a wave. As a wave, one can imagine "clouds" of electron orbitals around an atom, which are not physical things but rather representations of probabilities.

Conversely, electrons display properties that normally result from an extended structure, namely, angular momentum (spin), a magnetic moment, and some sort of internal oscillation.

The point-like depiction of elementary particles is so unsatisfactory that it has spawned new theories of matter known as $\mathrm{M}$ theories and quantum gravity.

In 1928, when Paul Dirac presented the wave function of the electron in the Dirac equation [2], it became obvious that there must be not only an internal oscillation but also some type of internal motion at the speed of light. Therefore, the seemingly empty space that surrounds electrons is made up of "virtual particles," and electrons are inseparable from the clouds of virtual particles surrounding them.

Moreover, there is no theory that quantifies particles in a meaningful way using appropriate calculations. This implies that quantum mechanics actually have no need for a particle as a concept because all the calculations are similar whether or not hard particles exist.

Subsequently, physicists attributed this intrinsic contradiction between the electron's different properties to the common-sense view that the electron is subject to quantum mechanics and, therefore, is not accessible to human imagination.

However, because subatomic particles can't yet be directly observed, scientists learn about the objects through indirect evidence. By observing what happens in the vacuum around negatively charged electrons-thought to be swarming with clouds of as-yet-unseen particles-researchers can create models of particle behavior.

The Standard Model predicts that particles surrounding electrons do affect an electron's shape, and according to this framework, the electron should be close to perfectly spherical. But at such an infinitesimal scale as to be pretty much undetectable using existing technology. One framework to explain physics beyond 
the Standard Model is known as supersymmetry. However, this theory predicts that the electron has a more distorted shape than that suggested by the Standard Model. According to this idea, the electron is predicted to be slightly aspheric [3].

In particle physics, the fundamental blocks of matter are continuous fluid-like substances known as "quantum fields" that permeate the whole space around us.

With this article, the vacuum is proposed to behave as a superfluid and a vortex shape of the electron is proposed as a condensation of the vacuum.

Hydrodynamics laws are applied to the superfluid vacuum to describe the basic properties of the electron. Hydrodynamic formulas are provided to calculate its mass, density, volume, time, constant angular momentum (spin), and electric charge.

\section{Nature of the Vacuum}

Both dark matter and new subatomic particles that were not predicted by the Standard Model are yet to be directly spotted; still, a growing amount of compelling evidence suggests that these phenomena do exist.

Vacuum density is generally viewed as a fundamental property of the cosmos whose magnitude should not depend on whether we choose subatomic, astronomical, or cosmological methods to assess its value.

According to quantum field theory, even in the absence of real particles, the vacuum is always filled by pairs of created and annihilated virtual particles. Therefore, the physical vacuum is assumed to be a non-trivial medium to which one can associate a certain energy and density. Therefore, quantum theory requires additional attributes to the vacuum. For instance, vacuum is not empty as previously considered but rather filled with quantum mechanical zero point energy.

The simulation of gravity (as far as Newton's universal law of gravitation is concerned) run through a computational fluid dynamic (CFD) approach is proven successful [4] and it seems that such an approach (space quanta absorption affected by massive particles, described as vortices of the same quanta [4]) is also able to describe any other effect related to general relativity's space topology.

In superfluid vacuum theory, the physical vacuum is described as a quantum superfluid and is characterized to behave like a frictionless fluid with density and extremely high thermal conductivity. The vacuum extends everywhere, has no size, shape, center, direction, time, or extent, and is immovable.

Superfluid vacuum theory proposes a mass generation mechanism that may replace or supplement the electroweak Higgs theory. It has been shown that the masses of elementary particles could be a result of interactions with a superfluid vacuum, similar to the gap generation mechanism in superconductors [5] [6].

Therefore, vacuum energy has real physically observable consequences, and its properties can be observed as real physical effects [7] [8].

The quantization of gravity indicates that there is an elementary quantum of 
matter, indivisible, whose virtual imaginary mass is $m_{0 i(\min )}= \pm 3.9 \times 10^{-73} \mathrm{~kg}$ [9].

These elementary quanta of matter should fill all the space in the Universe, forming a continuous and stationary quantum fluid. The density of the universal quantum fluid is clearly not uniform throughout the Universe because it can be strongly compressed in several regions (e.g., galaxies, stars, black holes, and planets). In the normal state (free space), the above-mentioned fluid is invisible.

In the super-compressed state, it can become visible in the form of known matter, since matter, as we have seen, is quantized and is consequently formed by an integer number of imaginary elementary quanta of matter with imaginary

mass $m_{0 i(\min )}$. This means that there are no particles in the Universe with masses smaller than the minimum mass and that all bodies are formed by a whole number of these particles [10].

\section{Internal Structure of the Electron}

The Standard Model describes most of the interactions between all of matter's building blocks, as well as the forces that act on those particles. For decades, this theory has successfully predicted how matter behaves, however it does not predict the structure of the electron.

The angular momentum (spin) of the electron indicates that there is an internal rotation that confers upon it its rest mass. According to Higgs theory, the interaction between particles and the Higgs field is continuously maintained and renewed, converting the amorphous potential energy of the field into individual structures. The seemingly empty space that surrounds the electron is teeming with pairs of particles and antiparticles that fleet in and out of existence; these are called "virtual particles".

Although precisely measuring this cloud is beyond the capabilities of modern methods, the current model predicts that electrons are slightly aspheric, with a distortion characterized by the electric dipole moment. However, no experiment so far has detected this deviation [11]. Thus, the electric dipole moment remains an elusive (and unproven) phenomenon, in which an electron's spherical shape appears deformed- "dented on one end and bulged on the other" [12].

We propose that the electron is an irrotational circular vortex of frictionless superfluid space with concentric streamlines that was created from the primordial vacuum during the Big Bang. The rate of rotation of the fluid is greatest at the center and decreases progressively with distance from the center until there is no gradient pressure on the boundaries of the vortex where the flow is laminar and the friction is null. In such a case, the absence of friction would make it impossible to create or destroy the vortex motion. If the negative suction point volume in the center of the vortex does not have enough energy to drag the virtual photons to the speed of light, then a stable situation cannot occur [13].

If we take a deeper look at the spiral arms of the electron vortex, we notice that the currents are made of smaller vortices that correspond to the Higgs par- 
ticles. Higgs particles have no mass when they are created but acquire mass when they travel through space. The interaction between the whirlpool-shaped particles and the Higgs field (the vacuum) is continuously maintained and renewed, converting the amorphous potential energy of the field into Higgs particle vortices.

The superfluid accommodates the rotation by forming a lattice of quantized vortices in which the vortex core, typically singular, breaks the topological constraint against rotational motion.

This new viewpoint of particles allows us to see them as a composite web structure and at the same time as energy motion processes (Figure 1).

A spinning system along an axis with angular momentum has a torque when the force is directed toward the center of gravity; this is known as the Coriolis effect.

The flow to the center of the vortex due to the Coriolis effect results in vortex tubes, which are always composed of the same virtual particles that rotate at the speed of light; they remain unbroken, so they are ring-like (Figure 2).

If the velocity of the space circulation reaches the limiting speed, $c$, which is the speed of light in the absolute vacuum, and the velocity field gradient around the center of the vortex becomes the postulated limiting angular rotation, $\omega$, the

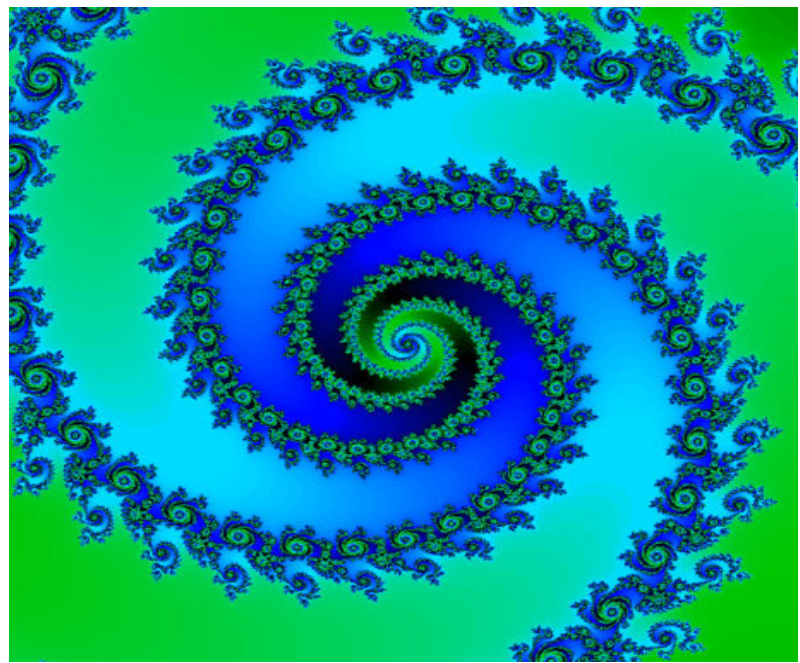

Figure 1. Artistic presentation of an elementary particle that has a vortex structure made by mini vortices of Higgs bosons.

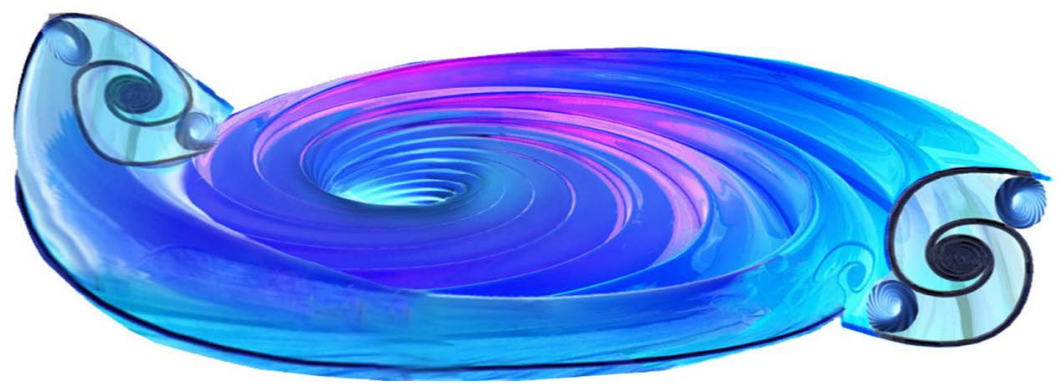

Figure 2. Two vortex tube constituents of an electron vortex. 
space breaks down, creating a spherical void, which is defined as a field-less, energy-less, and space-less volume of vacuum at the vortex center.

These maximums occur at the point where the centrifugal force and the radial force are equalized. The inflowing medium and the free surface dip sharply and the inflowing medium turns at $90^{\circ}$ near the axis line, with the depth and velocity inversely proportional to $r^{2}$, to form a concave paraboloid. This is where the highest vortex energy exists [14].

Maxwell worked out a theory of electromagnetism assuming that every magnetic tube of force was a vortex with an axis of rotation coinciding with the direction of the force. Several properties have been mathematically proved for a perfect frictionless fluid [15].

The magnitude of the vorticity in a vortex line increases proportionally as the vortex line is stretched. Consider a very thin vortex tube around the vortex line, so thin that the vorticity is practically constant over its width. As the vortex tube stretches, the cross-sectional area decreases by the same factor; thus, the vorticity must increase proportionally for the flux across the cross section to remain constant.

Therefore, the electron is a vortex characterized by its power and volume, with the specific magnitude depending on the rest energy of the electron.

\section{Mass of the Electron Vortex}

The angular momentum (spin) indicates that there is an internal rotation that determines the rest mass. The mass of an electron is the amount of fluid-like virtual photons with a certain density that passes in $1 \mathrm{~s}$. Therefore, the mass of the electron is calculated to be density times volume.

In hydrodynamics, the force $F$ that moves the vortex is directly related to the pressure that creates the vortex, known as the dynamic pressure $P_{d}$ and the area $A$ according to the formula

$$
F=P_{d} A .
$$

The dynamic pressure $\left(P_{d}\right)$ representing the fluid kinetic energy is expressed as

$$
P_{d}=\frac{1}{2} \rho v^{2},
$$

where $\rho$ is the density of the fluid and $v=c$ is the velocity. Therefore,

$$
F=\frac{1}{2} \rho c^{2} A .
$$

The area of the vortex is approximately a circle, $A=2 \pi r^{2}$, that interacts with the adjacent vacuum from both sides; therefore,

$$
F=\rho c^{2} \pi r^{2} .
$$

If we multiply and divide the right side of the above equation by time $t$, we obtain 


$$
F=\rho c t \pi r^{2} c / t .
$$

However, $v / t$ is equivalent to the acceleration $a$, and $v t$ is equivalent to the length $L$.

According to Newtonian theory, the force $F$ divided by the acceleration equals the mass. Dividing both sides by the acceleration, we obtain

$$
F / a=m=\rho \pi r^{2} L .
$$

However, the area times the length is equal to the volume $Q$. Therefore,

$$
F / a=m=\rho \pi r^{2} L .
$$

which corresponds to the inertial mass of the electron.

\section{Minimum Time of the Electron Vortex}

Because the electron has no shape in quantum physics, the time of the electron has never been reported before. The electron as a vortex has a minimum time less than which the electron converts into a virtual particle.

If $m=\rho \pi r^{2} L$ and the length is the product of velocity times time, $L=c t$, then

$$
m=\rho \pi r^{2} t c .
$$

The mass of the electron depends on the spin and the time. If the relative rotation velocity of the vortex or time is zero, the mass will be zero; accordingly, the mass will disappear and convert into an amorphous vacuum. The minimum time of the electron, that needed to complete one rotation cycle $2 \pi r_{e}$, is

$$
t=2 \pi r_{e} / c=8.08586 \times 10^{-21} \mathrm{~s},
$$

where $r_{e}=3.86 \times 10^{-13} \mathrm{~m}$ and $c=2.99792458 \times 10^{8} \mathrm{~m} / \mathrm{s}$.

\section{Density and Volume of the Electron}

Normally, electron density is a measure of the probability of an electron being present at a specific location. However, what we mean by the density of an electron is the density of virtual photons of which the electron is made. Hydrodynamically, vortex formation depends on the density of the medium in which it occurs. In our case, the vacuum's "energy density" is related to the number of virtual particles that are continually created and annihilated in the vacuum; however, the density of the electron is in a supercompressed state and is higher than that of the surrounding vacuum field.

Knowing the time of the electron, the density of the electron can be calculated:

$$
\rho=m / \pi r^{2} t c=2.519470 \times 10^{6} \mathrm{~kg} / \mathrm{m}^{3},
$$

where $\pi r^{2} t c$ is the volume. Knowing the density of the electron, the volume $V_{e}$ can be calculated:

$$
V_{e}=m / \rho=3.6118 \times 10^{-37} \mathrm{~kg} / \mathrm{m}^{3} .
$$




\section{Particle-Wave Relationship}

Particle-wave duality is a central tenet of quantum physics, and an electron has wave-like properties. The essence and physical relationship between the particle and the wave remains an unresolved problem in physics. The vortex model of the electron gives three different solutions:

1) Louis de Broglie developed a hypothesis [16] to relate the dual wave and particle behavior that can be applied to electrons. The de Broglie wavelength formula relates the wavelength $\lambda$ to the momentum $m v$ of a wave/particle: [17]

$$
\lambda=h / p=h / m c \text { and } h=\lambda m c .
$$

In hydrodynamics, the velocity of the fluid element instantaneously passing through a given point in space in the vortex with radius $r$ is constant in time; therefore, the circulation or the vorticity in the core of the vortex $2 \pi r c=\Gamma$ is constant. $\Gamma \mathrm{m}$ is a conserved momentum; therefore, $2 \pi r c m$ is constant, which corresponds to the Planck constant.

If $h=2 \pi r \mathrm{rm}$, then $\lambda=2 \pi r$, which is the circumference of the core of the vortex.

If the radius of the core of the vortex electron,

$$
r=h / 2 \pi m c=3.86 \times 10^{-13} \mathrm{~m}
$$

where $m$ is the mass of the electron, the value of the circumference is $2 \pi r=2.42408 \times 10^{-12} \mathrm{~m}$, which is similar to the CODATA 2014 [18] value for the Compton wavelength of the electron, $2.4263102367(11) \times 10^{-12} \mathrm{~m}$.

Vortices also have frequencies described in units of time (rotations per second). If the time required to complete a cycle around the vortex is $t=2 \pi r_{e} / c=8.08586 \times 10^{-21} \mathrm{~s}$, then the frequency of the electron related to the cycles of rotation of the vortex is $f_{e}=1 / t=1.2367 \times 10^{20} \mathrm{~Hz}$. In fact, the rotational velocity of the vortex $\omega=2 \pi f=c / r$; therefore, $f=c / 2 \pi r$, which gives us the same frequency as Planck's theory. If the invariant mass of an electron is approximately $9.109 \times 10^{-31}$ kilograms, then its energy would be $E=m c^{2}=8.1981 \times 10^{-14} \mathrm{~J}$

And if $E=h f$ where $h$ is Planck constant $=6.62607004 \times 10^{-34} \mathrm{~m}^{2} \cdot \mathrm{kg} / \mathrm{s}$ then $f=E / h=1.2372492 \times 10^{20} \mathrm{~Hz}$.

2) The third solution is related to the energy of the particle. A particle of mass $m$ has a rest energy of $E=m c^{2}$. The non-reduced Compton wavelength for this particle is the wavelength of a photon of the same energy. According to Planck's theory, for photons of frequency $f$, the energy is given by

$$
E=h f_{e} .
$$

The frequency of the electron is

$$
f_{e}=E / h,
$$

where $f_{e}=E / h=0.511 \mathrm{MeV} / h=8.1866 \times 10^{-7} \mathrm{ergs} / h$. Therefore,

$$
f_{e}=1.2355 \times 10^{20} \text { cycles } / \mathrm{s} \text {. }
$$


where $h$ is $6.626176 \times 10^{-27}$ erg-seconds.

How is $E=h f$ related to the vortex model?

The force that rotates the vortex is

$$
F=\rho c^{2} \pi r^{2} .
$$

If we multiply and divide the right side of Equation (3.5.3) by time, $t$, we obtain

$$
F=\rho c t \pi r^{2} c / t,
$$

where $c t$ is equivalent to the distance $L, L \pi r^{2}$ is equivalent to the volume $Q, \rho Q$ is equivalent to the mass, and $1 / t$ is equivalent to the frequency $f$.

Therefore,

$$
F=m c f .
$$

If $E=$ force $\times$ distance and the distance that an electron moves in one cycle is $2 \pi r$, then $E=2 \pi r m c f$, where $2 \pi r m c$ is equivalent to $h$. Therefore, $E=h f$ and $f_{e}=E / h$.

\section{Electron Charge}

Charge is a fundamental physical property of matter that is responsible for its interactions with electromagnetic fields. An electron is a particle that possesses this property, and experiments show that it possesses a negative charge.

The real nature and the essence of charge are unknown. In this section, a new theory to describe the nature of electric charge is formulated based on the vortex model of the electron.

In hydrodynamics, the rotation of a vortex creates a drag force that attracts the vacuum to the center of the vortex. This force is directly related to the density of the vacuum, the speed of rotation, and the area is inversely related to the distance from the vortex center according to the equation

$$
F=\frac{1}{2} \rho c^{2} A / r .
$$

Multiplying and dividing the right side of the above equation by time, we obtain

$$
F=\frac{1}{2} \rho c t A c / r t=\frac{1}{2} \rho V c / r t .
$$

However, this force is reduced owing to interactions with the adjacent vacuum. If the density of the vacuum is $\rho_{v}$ and the rotation speed of the vortex is $c$, dividing the momentum by the length of the circumference of the vortex will give the diminished momentum $P$ for a unit of length:

$$
P=\rho_{v} c / \lambda .
$$

Therefore, the momentum of the vortex is reduced for every unit of length according to the equation

$$
F=\frac{1}{2} \rho V \rho_{v} c^{2} / r t \lambda
$$


If $\lambda=2 \pi r$, then

$$
F=\frac{1}{2} \rho V \rho_{v} c^{2} / r t 2 \pi r=\rho V \rho_{v} c^{2} / 4 t 2 \pi r^{2} .
$$

In hydrodynamics, $\rho_{v} c^{2}$ is the elasticity of the vacuum. In fact, the velocity of a particle in an elastic medium can be expressed by the formula

$$
c=(E / d)^{1 / 2}
$$

where $c$, the speed of light, $E$ is the elasticity, and $d=\rho_{r}$, the density of the medium.

Therefore, the elasticity, $E$, can be written as

$$
E=\rho_{v} c^{2}
$$

which is the inverse of the stiffness of the vacuum and has the same value as the electric permittivity, i.e., $\rho_{v} v^{2}=\varepsilon_{0}^{-1}$.

This makes sense in terms of dimensions because the elasticity modulus is Newton $\times \mathrm{m}^{-2}$ whereas the permittivity is Newton ${ }^{-1} \times \mathrm{m}^{-2}\left(\mathrm{C}^{2} / \mathrm{Nm}^{2}\right)$.

Then, the Equation (3.6.5) becomes

$$
F=\rho_{v} V / 4 t \pi r^{2} \varepsilon_{0}
$$

The force density at a point in a fluid divided by the density is the acceleration of the fluid at that point:

$$
F / \rho=f=V / t 4 \pi r^{2} \varepsilon_{0} .
$$

In fluid mechanics, the force density [19] is the negative gradient of the pressure. It has physical dimensions of force per unit volume. The force density is a vector field representing the flux density of the hydrostatic force within the bulk of a fluid.

Furthermore, in fluid dynamics, the volume of a fluid that passes per unit of time, $V / t$, is the volume flow rate, which is usually represented by the symbol $Q$. Its SI unit is $\mathrm{m}^{3} / \mathrm{s}$.

The force between two vortices is directly proportional to the magnitude of the flow rate of the elementary density in each vortex $q_{1} q_{2}$ and inversely proportional to the distance of the separation between their centers, $r$, diminished by the stiffness of the vacuum represented by the electric permittivity per unit of length:

$$
f=q_{1} q_{2} / 4 \pi \varepsilon_{0} r^{2}
$$

The origin and essence of the electric permittivity will be discussed in detail separately in another paper.

\section{Discussion}

The nature and the essence of the electron was central to the development of quantum theory early in the twentieth century, and remains at the frontier of physics today. A century after Danish physicist Niels Bohr conceived of the electron as the proton's satellite [20], our perception of the electron continues to 
evolve and expand.

In particle physics, the fundamental blocks of matter are continuous fluid-like substances known as "quantum fields" that permeate the whole space around us.

In this article, the electron is proposed to have frictionless vortex shape and the hydrodynamic laws are applied.

The central idea is that an electron is a frictionless vortex with conserved momentum made out of condensed vacuum generated in the Big Bang from massless virtual photons that acquire mass when moving in the vortex at the speed of light, as described by Higgs theory. Considering the vacuum density and applying classical hydrodynamics, analytical formulations are applied to calculate the mass, volume, density, time, and frequency of the vortex. We obtained the properties of an electron and unified particle-wave duality using the same vortex model.

We summarize the findings of this article in the following points:

1) The electron has a vortex shape with density, mass, radius, area, circumference, volume, rotational velocity, minimal time, frequency and flow rate.

2) The force that rotates the vortex is directly proportional to the density of the vortex, times the square of its rotation speed and to its area according to the Equation (4) $F=\rho c^{2} \pi r^{2}$.

3) The electron vortex mass is directly proportional to its density times its area and its length according to the Equation (6) $m=\rho \pi r^{2} L$.

4) The density of the electron is directly proportional to the vortex mass and inversely proportional to its area, and to the time needed to complete one cycle and rotation speed of the vortex according to the Equation (10) $\rho=m / \pi r^{2} t c$.

5) The minimum time of the electron is the time needed to complete on rotation cycle of the vortex which directly proportional to the vortex circumference and inversely to the rotation speed according to the Equation (9) $t=2 \pi r_{e} / c$.

6) Therefore, the frequency of the electron is directly proportional to its rotation speed and inversely related to its circumference according to the equation in section (6.1) $t=2 \pi r_{e} / c$.

7) Furthermore, the frequency of the electron vortex is directly related to its energy and inversely related to its mass, circumference and rotation speed according to the equation in section (6.2) $f=E / 2 \pi r m c$.

8) The electric charge $q$ is equal to flow rate of the elementary density $V / t$ from the periphery to the center of the electron vortex divided by the area in both sides of the vortex diminished by the stiffness of the vacuum according to the Equation (25) $f=V / t 4 \pi \varepsilon_{0} r^{2}=q / 4 \pi \varepsilon_{0} r^{2}$.

9) The electric force between two charges is the force between two vortices which is directly proportional to the magnitude of the flow rate of the elementary density in each vortex and inversely proportional to the distance of the separation between their centers, diminished by the stiffness of the vacuum represented by the electric permittivity per unit of length according to the Equation (26) $f=q_{1} q_{2} / 4 \pi \varepsilon_{0} r^{2}$. 
We conclude that the shape of the electron is accessible to human imagination.

The electron properties can be accurately described using classical laws of hydrodynamics. Knowing its shape helps to determine its properties and shed a light on how matter is made and to explain the interactions of sub-atomic particles.

This theory could overturn several alternative physics theories that attempt to fill in the blanks about phenomena that the Standard Model can't explain.

The electron wave-particle duality, electron charge and the origin of electron spin will be discussed in detail in separate papers.

Future experimental studies are needed to confirm the vortex structure of the electron.

\section{Acknowledgements}

The author would like to thank Enago (https://www.enago.com/) for the English language review.

This research did not receive any specific grant from funding agencies in the public, commercial, or not-for-profit sectors.

\section{Conflicts of Interest}

The author declares no conflicts of interest regarding the publication of this paper.

\section{References}

[1] Braibant, S., Giacomelli, G. and Spurio, M. (2012) Particles and Fundamental Interactions: An Introduction to Particle Physics. 2nd Edition, Springer, Berlin, 1-3. https://doi.org/10.1007/978-94-007-2464-8

[2] Dirac, P.A.M. (1982) Principles of Quantum Mechanics. International Series of Monographs on Physics. 4th Edition, Oxford University Press, Oxford, p. 255.

[3] Khriplovich, I.B. and Lamoreaux, S.K. (1997) CP Violation without Strangeness. Springer, New York. https://doi.org/10.1007/978-3-642-60838-4

[4] Fedi, M. (2015) Hypothetical Role of Quantum Space-Time's Superfluid Dynamics in the Physics of Particles and Fundamental Interactions. https://hal.archives-ouvertes.fr/hal-01223102

[5] Zloshchastiev, K.G. (2011) Spontaneous Symmetry Breaking and Mass Generation as Built-in Phenomena in Logarithmic Nonlinear Quantum Theory. Acta Physica Polonica B, 42, 261-292.

[6] Avdeenkov, A.V. and Zloshchastiev, K.G. (2011) Quantum Bose Liquids with Logarithmic Nonlinearity: Self-Sustainability and Emergence of Spatial Extent. Journal of Physics B: Atomic, Molecular and Optical Physics, 44, Article ID: 195303. https://doi.org/10.1088/0953-4075/44/19/195303

[7] Rauscher, E.A. (1968) Electron Interactions and Quantum Plasma Physics. Journal of Plasma Physics, 2, 517-541.

[8] Rauscher, E.A. (2004) Dynamic Plasma Excitation Modes of Propagation in the Ionosphere. PA Press, Wisconsin, 13, 295. 
[9] De Aquino, F. (2010) Mathematical Foundations of the Relativistic Theory of Quantum Gravity. Pacific Journal of Science and Technology, 11, 173-232.

[10] De Aquino, F. (2012) The Universal Quantum Fluid, in the Theory of Everything.

[11] Hudson, J.J., Kara, D.F.M., Smallman, I.J., Sauer, B.E., Tarbutt, M.R. and Hinds, E.A. (2011) Improved Measurement of the Shape of the Electron. Nature, 473, 493-496. https://doi.org/10.1038/nature10104

[12] Weisberger, M. and LiveScience (2018) Measurement Shows the Electron's Stubborn Roundness. Scientific American October 18, 2018.

https://www.scientificamerican.com/article/measurement-shows-the-electrons-stub born-roundness

[13] Winterberg, F. (2001) Planck Mass Rotons as Cold Dark Matter and Quintessence. 9th Canadian Conference on General Relativity and Relativistic Astrophysics, Edmonton, 24-26 May 2001.

[14] http://www.softcom.net/users/greebo/vortex.htm

[15] Maxwell, J.C. (1861) On Physical Lines of Force. Philosophical Magazine, 90, 11-23. https://doi.org/10.1080/14786431003659180

[16] Feynman, R. (1990) QED the Strange Theory of Light and Matter. Penguin Edition, p. 84.

[17] de Broglie, L. (1923) Radiations-Ondes et quanta" [Radiation-Waves and Quanta]. Comptes Rendus (in French), 177, 507-510+548.

[18] CODATA 20104 Value for Compton Wavelength for the Electron from NIST.

[19] Density, F. (2012) Eric Weisstein's World of Physics.

[20] Bohr, N. (1913) Two New Noctuids from French Guiana. Philosophical Magazine, 26, 1-25. https://doi.org/10.5962/bhl.part.9556 